\title{
Application of multiset theory for the selection of the single result from alternatives aggregate obtained using different decision methods
}

\author{
Denis Solovjev ${ }^{1,2, *}$, Inna Solovjeva ${ }^{2}$, and Yuri Litovka ${ }^{2}$ \\ ${ }^{1}$ Tambov State University named after G.R. Derzhavin, 392000, Internatsionalnaya, 33, Tambov, \\ Russia \\ ${ }^{2}$ Tambov State Technical University, 392000, Sovetskaya, 106, Tambov, Russia
}

\begin{abstract}
In this article are discussed the main reasons to obtain different results in decision-making by various methods. It is proposed to use the multiset theory for selection a single result from a solution aggregate. In the work is described mathematical decision-making model based on the argument calculating for maximizing the elements multiplicity functions of the arithmetic sum to found multiset solutions. Here are presented decisions compare results, which were made by expert group and according to the approach proposed by the authors on an example of the galvanic coating choice. In this article are represented recommendations for improving the efficiency of the proposed approach for obtaining the unit dimension of the resulting multiset solutions.
\end{abstract}

\section{Introduction}

The solution of applied problems with using of computer systems becomes the traditional approach to make decision. The by computer systems found solutions depend directly on the completeness and consistency of the data, the method of processing them, and the high dimensionality of the solution space [1]. The solution of the same problem by various methods may be different due to the subjectivity inherent in the methods of data processing by computer systems. To select a single result from a set of such solutions is proposed to use the principle of building reliable machines from unreliable elements proposed by J. von Neumann in [2]. According to this principle, to make a decision, is introduced redundancy of decisions made using several different methods. The choice of a single solution in this case will be carried out on the majority of coinciding solutions using the multiset theory [3].

The aim of the work is to applicate of the multiset theory for the selection of the single result from alternatives aggregate obtained using different decision methods.

\footnotetext{
*Corresponding author: solovjevdenis@mail.ru
} 


\section{Mathematical model of decision-making using multisets}

The solution of the problem with using of the multiset theory is carried out as follows.

There is a domain set $\mathbf{D}_{1}$ of facts, criteria, etc., which contains the current information for the problem under consideration:

$$
\mathbf{D}_{1}=\left\{a_{1}, a_{2}, a_{3}, \ldots\right\} .
$$

There is also a domain set $\mathbf{D}_{2}$ of alternatives to solve the problem:

$$
\mathbf{D}_{2}=\left\{b_{1}, b_{2}, b_{3}, \ldots\right\} .
$$

Let the decision on the considered problem be made on the basis of $N$ methods. The set of required information $\mathbf{X}_{i}$ is formed for each method:

$$
\mathbf{X}_{i}=\left\{x_{i} \mid x_{i} \in \mathbf{D}_{1}\right\} .
$$

The multiset $\mathbf{Y}_{i}$ of the decisions made over the set $\mathbf{X}_{i}$ is obtained as a result to application of the $f_{i}$ method:

$$
f_{i}: \mathbf{X}_{i} \rightarrow \mathbf{Y}_{i}, i=1, \ldots, N
$$

which has the following form:

$$
\mathbf{Y}_{i}=\left\{k_{\mathbf{Y}_{i}}\left(y_{i}\right) \cdot y_{i} \mid y_{i} \in \mathbf{D}_{2}, k_{\mathbf{Y}_{i}}\left(y_{i}\right) \in \mathbf{Z}_{+}\right\},
$$

where $k_{\mathbf{Y}_{i}}\left(y_{i}\right) \geq 0$ is multiplicity function which specifies the number of repetitions of $y_{i}$ elements in the $\mathbf{Y}_{i}$ decision; $\mathbf{Z}_{+}=\{0,1,2, \ldots\}$ is the set of non-negative integers.

Then let us search the arithmetic sum of $N$ obtained multisets (5) by summing the multiplicity function values of the corresponding elements that are present in at least one of the multisets:

$$
\sum_{i=1}^{N} \mathbf{Y}_{i}=\left\{k_{\Sigma}\left(y_{i}\right) \cdot y_{i} \mid k_{\Sigma}\left(y_{i}\right)=\sum_{i=1}^{N} k_{\mathbf{Y}_{i}}\left(y_{i}\right)\right\} .
$$

The height of the arithmetic sum of multisets (6) is calculated, which is equal to the maximum value $k_{\Sigma}$. The final set of $\mathbf{Y}^{*}$ decisions are formed from those peak elements $y^{*}$, the multiplicity function for which coincides with the height:

$$
\mathbf{Y}^{*}=\underset{y^{*} \in \mathbf{D}_{2}}{\arg \max } \sum_{i=1}^{N} \mathbf{Y}_{i}=\left\{k_{\max }\left(y^{*}\right) \cdot y^{*} \mid k_{\max }\left(y^{*}\right)=\max _{y_{i} \in \mathbf{D}_{2}} k_{\Sigma}\left(y_{i}\right)\right\} .
$$

Let us calculate the dimension of the resulting set (7) by counting the total number of different elements of $y^{*}$ :

$$
/ \mathbf{Y}^{*} /=\sum_{y^{*} \in \mathbf{D}_{2}} \chi_{\mathbf{Y}^{*}}\left(y^{*}\right)
$$

where $\chi_{\mathbf{Y}^{*}}\left(y^{*}\right)$ is characteristic function which takes the following values: 


$$
\chi_{\mathbf{Y}^{*}}\left(y^{*}\right)=\left\{\begin{array}{l}
1, \text { if } y^{*} \in \mathbf{Y}^{*} \\
0, \text { otherwise }
\end{array} .\right.
$$

The value of the dimension $/ \mathbf{Y}^{*} /=0$ indicates that there are no alternative. Lack of information may be caused by a lack of awareness on the part of the decision-maker or by a lack of time to check the empirical basis of possible solutions. The value of the dimension $/ \mathbf{Y}^{*} />1$ shows that there are among the alternatives there are no dominant state solutions, i.e. different alternatives correspond to the best result in different condition of the problem being solved. The choice of a single alternative is not possible in both cases and the domain sets $\mathbf{D}_{2}$ or $\mathbf{D}_{1}$ must be corrected for $/ \mathbf{Y}^{*} /=0$ or $/ \mathbf{Y}^{*} />1$ respectively.

\section{Materials and methods}

To compare the results of the decision with using of multiset theory is carried out according to the approach shown on fig. 1.

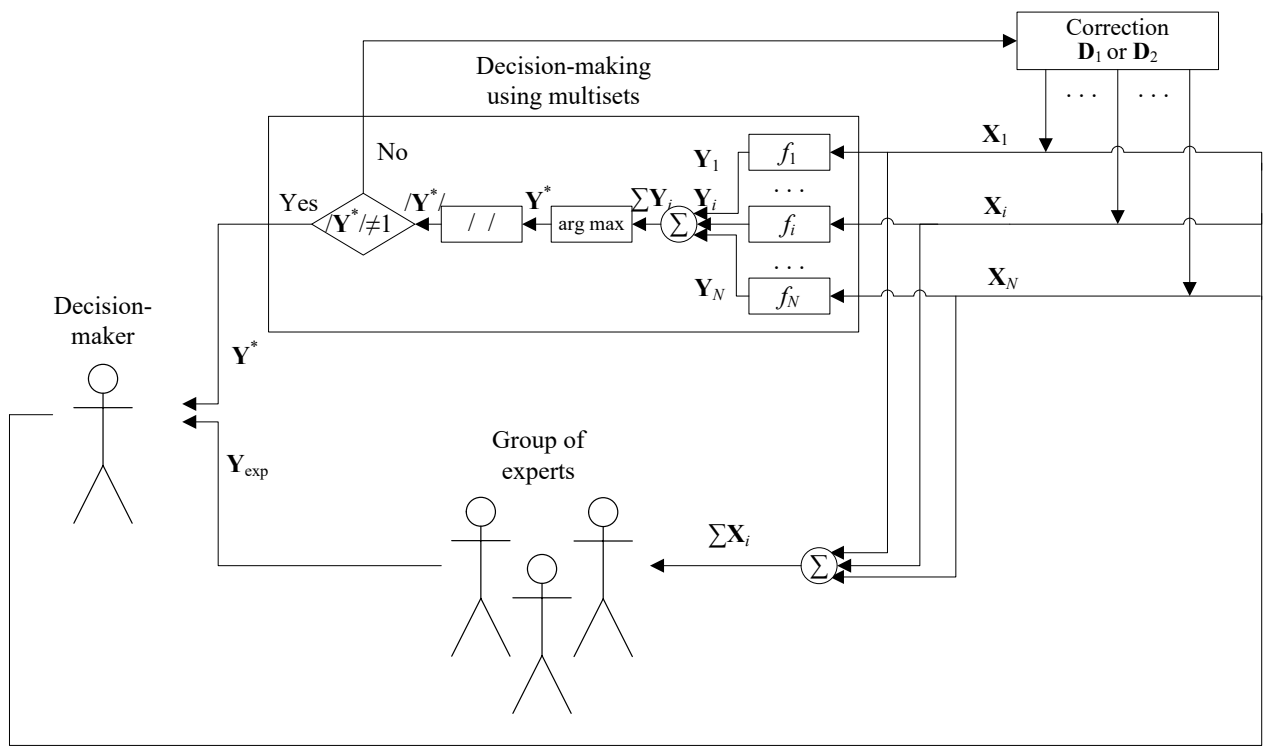

Fig. 1. Block diagram for result comparison of decision-making on the basis of multisets and expert group

Let us consider the problem of decision-making on example of galvanic coating choice. As a comparative element is selected the following group of experts: process engineer, design engineer and technical control engineer. Decision-making by experts is carried out using a questionnaire by the Delphi method [4].

The domain set $\mathbf{D}_{1}$ contains the following information for the problem: $a_{1}-$ metal of part; $a_{2}$ - purpose of coating; $a_{3}$ - coating thickness; $a_{4}$ - climatic performance of the part; $a_{5}$ - placement category of parts; $a_{6}-$ additional instructions; $a_{7}-$ cost; $a_{8}-$ microhardness; $a_{9}$ - adhesion; $a_{10}$ - roughness; $a_{11}$ - porosity; $a_{12}$ - reflectivity; $a_{13}-$ geometric shape of a part; $a_{14}$ - batch volume; $a_{15}$ - environmental friendliness of the process; $a_{16}$ - process duration.

The domain set $\mathbf{D}_{2}$ of alternatives according to [5] looks like: $b_{1}-$ aluminum; $b_{2}$ - gold; $b_{3}$ - cadmium; $b_{4}-$ cobalt; $b_{5}-$ copper; $b_{6}-$ nickel; $b_{7}-$ tin; $b_{8}-$ palladium; $b_{9}-$ 
platinum; $b_{10}-$ rhodium; $b_{11}-$ lead; $b_{12}-$ silver; $b_{13}-$ titanium; $b_{14}-$ chromium; $b_{15}-$ zinc.

To solve the considered problem are chosen $N=3$ methods, among which: $f_{1}-$ GOST 9.303-84 [6]; $f_{2}$ - analytic hierarchy process, which is described in the works $[7,8] ; f_{3}-$ production model of knowledge, which is described in the works $[9,10]$.

The set of required $\mathbf{X}_{1}$ data for all experiments is the aggregate of $a_{1}-a_{6}$ elements for the considered $b_{1}-b_{15}$ alternatives. Various samples of elements from $\mathbf{D}_{1}$ and $\mathbf{D}_{2}$ were used for the sets $\mathbf{X}_{2}$ and $\mathbf{X}_{3}$.

The expert group adopts the decision of $\mathbf{Y}_{\exp }$ on the basis of the same information, which is available to all $\mathrm{N}$ methods in aggregate $\left(\sum \mathbf{X}_{i}\right)$.

\section{Results and discussion}

There were carried out 40 experiments to compare the result of decision making on the basis of multisets and the group of experts. The most interesting for the analysis results are shown in Table 1.

Table 1. The results of decision making.

\begin{tabular}{|c|c|c|c|c|c|c|c|c|c|}
\hline $\begin{array}{c}\text { Number of } \\
\text { experiments }\end{array}$ & $\mathbf{X}_{1}$ & $\mathbf{X}_{2}$ & $\mathbf{X}_{3}$ & $\mathbf{Y}_{1}$ & $\mathbf{Y}_{2}$ & $\mathbf{Y}_{3}$ & $\mathbf{Y}^{*}$ & $/ \mathbf{Y}^{*} /$ & $\mathbf{Y}_{\text {exp }}$ \\
\hline 1 & \multirow{5}{*}{$\begin{array}{l}\left\{a_{1},\right. \\
a_{2}, \\
a_{3}, \\
a_{4} \\
a_{5} \\
\left.a_{6}\right\}\end{array}$} & $\begin{array}{c}\left\{\mathbf{X}_{1}, a_{7},\right. \\
\left.a_{16}\right\}\end{array}$ & $\begin{array}{c}\left\{\mathbf{X}_{1}, a_{7}\right. \\
\left.a_{14}\right\}\end{array}$ & $\begin{array}{l}\left\{b_{6},\right. \\
b_{14}, \\
\left.b_{15}\right\}\end{array}$ & $\left\{b_{6}\right\}$ & $\begin{array}{l}\left\{b_{6},\right. \\
\left.b_{15}\right\}\end{array}$ & $\left\{b_{6}\right\}$ & 1 & $\left\{b_{6}\right\}$ \\
\hline 2 & & $\begin{array}{l}\left\{a_{1}, a_{2},\right. \\
\left.a_{3}, a_{7}\right\}\end{array}$ & $\mathbf{X}_{2}$ & $\begin{array}{l}\left\{b_{2},\right. \\
\left.b_{9}\right\}\end{array}$ & $\left\{b_{9}\right\}$ & $\left\{b_{2}\right\}$ & $\begin{array}{l}\left\{b_{2},\right. \\
\left.b_{9}\right\}\end{array}$ & 2 & $\left\{b_{8}\right\}$ \\
\hline 3 & & $\begin{array}{c}\left\{a_{3}, a_{4},\right. \\
a_{9}, a_{13}, \\
a_{14}, \\
\left.a_{15}\right\}\end{array}$ & $\begin{array}{c}\left\{a_{1}, a_{2},\right. \\
a_{3}, a_{4}, \\
a_{9}, a_{14}, \\
\left.a_{16}\right\}\end{array}$ & $\begin{array}{l}\left\{b_{6},\right. \\
\left.b_{15}\right\}\end{array}$ & $\left\{b_{6}\right\}$ & $\left\{b_{6}\right\}$ & $\left\{b_{6}\right\}$ & 1 & $\left\{b_{6}\right\}$ \\
\hline 4 & & $\begin{array}{c}\left\{a_{7}, a_{8},\right. \\
a_{9}, a_{10}, \\
a_{11}, \\
\left.a_{12}\right\}\end{array}$ & $\begin{array}{c}\left\{a_{13},\right. \\
a_{14}, a_{15}, \\
\left.a_{16}\right\}\end{array}$ & $\begin{array}{l}\left\{b_{3},\right. \\
\left.b_{5}\right\}\end{array}$ & $\left\{b_{15}\right\}$ & $\begin{array}{l}\left\{b_{6},\right. \\
\left.b_{7}\right\}\end{array}$ & $\begin{array}{c}\left\{b_{3},\right. \\
b_{5}, b_{6}, \\
b_{7}, \\
\left.b_{15}\right\}\end{array}$ & 5 & $\left\{b_{15}\right\}$ \\
\hline 5 & & $\begin{array}{c}\left\{a_{2}, a_{3},\right. \\
a_{4}, a_{8}, \\
\left.a_{9}, a_{13}\right\}\end{array}$ & $\mathbf{X}_{2}$ & $\begin{array}{l}\left\{b_{10}\right. \\
\left.b_{15}\right\}\end{array}$ & $\left\{b_{15}\right\}$ & $\begin{array}{l}\left\{b_{10},\right. \\
\left.b_{15}\right\}\end{array}$ & $\left\{b_{15}\right\}$ & 1 & $\left\{b_{15}\right\}$ \\
\hline
\end{tabular}

The value of the dimension $/ \mathbf{Y}^{*} />1$ was obtained in cases when: a) the sets of required data $\mathbf{X}_{i}$ for all $f_{i}$ had partially common elements (for example, the number of experiment 2 in Table 1); b) the sets of required data $\mathbf{X}_{i}$ for all $f_{i}$ did not contain common elements (for example, the number of experiment 4 in Table 1). If the most important data from $\mathbf{X}_{i}$ are present in all $N$ methods of $f_{i}$ decision-making, then in $90 \%$ of experiments the result of decision-making on the basis of set $\mathbf{Y}^{*}$ is the only and coincides with the opinion $\mathbf{Y}_{\exp }$ of the group of experts. The value of the dimension $/ \mathbf{Y}^{*} /=0$ was never obtained in the decision making process due to consideration of the maximum possible aggregate of elements from the domain set $\mathbf{D}_{2}$.

\section{Conclusion}

The conducted researches have shown that the choice of the single result from alternatives aggregate obtained using different decision methods can be achieved using the approach proposed by the authors. This approach is based on calculating the maximizing argument 
the functions of the arithmetic multiplicity elements sum of multisets from the solutions found. The number of methods should be at least 3 to apply the by the authors proposed approach. In turn, not so much numbers of methods can influence on the obtaining of the single problem solution, such as the sameness of the initial information and the space solution. Exceptions of several dominant alternatives, what are arising from decisionmaking by this or that method, can also be achieved by introducing weighting coefficients of competence for methods. Weighting coefficients of competence for each method are established by the person making the decision, in the interval $0 \ldots 1$. Then the value of the multiplicity function of the found solution for each method is multiplied by the value of the appropriate to him coefficient. It should be noted that one should not try to choose a large number of different methods of decision-making because of the complexity, duration and high cost of their implementation.

\section{References}

1. A.B. Petrovsky, Theory of decision making (Academy, Moscow, 2009)

2. J. von Neumann, Probabilistic logics and synthesis of reliable organisms from unreliable components, Automata Studies, v. 34, pp. 43-98 (1956)

3. A.B. Petrovsky, The spaces of sets and multisets (Editorial URSS, Moscow, 2003)

4. O. Helmer-Hirschberg, The Delphi method for systematizing judgments about the future, (University of California, Los Angeles, 1966)

5. Eh.B. Davidavichyus and etc., GOST 9.306-85 Unified system of corrosion and ageing protection. Metallic and non-metallic inorganic coatings. Symbols (Publishing house of standards, Moscow, 1987)

6. Eh.B. Davidavichyus and etc., GOST 9.303-84 Unified system of corrosion and ageing protection. Metal and non-metal inorganic coatings. General requirements for selection (Publishing house of standards, Moscow, 1985)

7. M.M. Kaoser, R.M. Mamunur, S. Ahmed, Selecting a material for an electroplating process using AHP and VIKOR multi attribute decision making method, International Conference on Industrial Engineering and Operations Management, pp. 834-841 (2014)

8. D.S. Soloviev, I.A. Mukina, Yu.V. Litovka, The solution of multicriteria problem of selection of galvanic coatings using the method of analytical hierarchy process, Vestnik of Astrakhan State Technical University. Series: Management, Computer Science and Informatics, v. 1, pp. 18-27 (2017)

9. L.A. Dobrzański, J. Madejski, Prototype of an expert system for selection of coatings for metals, Journal of Materials Processing Technology, v. 175(1), pp. 163-172 (2006)

10. I.A. Solovjeva, D.S. Solovjev, Yu. Litovka, Selection of the metal plating on the basis of the production model using the language CLIPS, On the way to the information society: a collection of articles of the International Scientific and Practical Conference, v. 1, pp. 424-426 (2017) 